\title{
A multi-frequency solution for the Burgers equation
}

\author{
Claes M. Hedberg \\ Department of Mechanical Engineering, University of Karlskrona-Ronneby \\ 37179 Karlskrona, Sweden \\ Internet: claes.hedberg@ima.hk-r.se
}

\begin{abstract}
A generalization of the single frequency Cole-Mendousse solution for the Burgers equation is shown. The solution is in the same form - a ratio between two Fourier series containing Bessel functions. The input is given as an arbitrary number of frequency components which can have any amplitude, frequency and phase. The solution is valid for any distance.
\end{abstract}

\section{INTRODUCTION}

The solution for multiple frequency conditions for the Burgers equation, which describes nonlinear propagation of plane waves through a dissipative medium, has been developed by Hedberg [1]. It is a generalization of the well known ColeMendousse solution for a single frequency source [2] [3]. Both of these are in a form of a ratio between Fourier series with Bessel function coefficients.

The only earlier multi-frequency solution is the one by Fenlon [4] [5]. The Fenlon solution does not take dissipation into account and is valid only before shock formation.

\section{SOLUTION FOR MULTIPLE FREQUENCIES}

The nonlinear equation for plane waves in a homogeneous dissipative medium is Burgers' equation, given in dimensionless variables as

$$
\frac{\partial V}{\partial \sigma}-V \frac{\partial V}{\partial \theta}-\epsilon \frac{\partial^{2} V}{\partial \theta^{2}}=0
$$

The definition of the dimensionless variables - using: a characteristic velocity $v_{0}$ of the medium, $b$ the effect of viscosity and heat conduction, $\tau=t-\frac{x}{c_{0}}$ the retarded time, $\rho_{0}$ the undisturbed density, $c_{0}$ the undisturbed sound velocity, $\beta=\frac{\gamma+1}{2}$ the nonlinearity for a fluid and a characteristic time $\frac{1}{\omega}$ - are 


$$
\begin{array}{clrl}
V=\frac{v}{v_{0}} & \theta=\omega \tau \\
\sigma=\frac{\beta}{c_{0}^{2}} \omega v_{0} x & \epsilon=\frac{1}{2 \beta} \frac{b \omega}{c_{0} v_{0} \rho_{0}}
\end{array}
$$

Let a boundary condition consisting of a number of $L$ frequencies be expressed as (the $\alpha_{l} \mathrm{~s}$ are integers):

$$
V(\sigma=0, \theta)=\sum_{l=1}^{L} a_{l} \sin \left(\alpha_{l} \theta+\gamma_{l}\right)
$$

The resulting solution to Burgers' equation (1) for this boundary condition, valid for any number of frequencies with arbitrary amplitudes, is

$$
V(\sigma, \theta)=i 2 \epsilon \frac{\sum_{k=-\infty}^{\infty} k c_{k} \mathrm{e}^{-k^{2} \epsilon \sigma} \mathrm{e}^{i k \theta}}{\sum_{k=-\infty}^{\infty} c_{k} \mathrm{e}^{-k^{2} \epsilon \sigma} \mathrm{e}^{i k \theta}}
$$

where, with $I_{n}$ being modified Bessel functions, the coefficients are

$$
c_{k}=\sum_{k=\sum n^{(l)} \alpha_{l}} I_{n^{(1)}}\left(\frac{a_{1}}{2 \alpha_{1} \epsilon}\right)(-1)^{n^{(1)}} \exp \left(i n^{(1)} \gamma_{1}\right) \ldots I_{n^{(L)}}\left(\frac{a_{L}}{2 \alpha_{L} \epsilon}\right)(-1)^{n^{(L)}} \exp \left(i n^{(L)} \gamma_{L}\right)
$$

This solution is a generalization of the Cole-Mendousse solution [2] [3]. It yields the Cole-Mendousse solution when the input is a single sine wave.

\section{NUMERICAL EXAMPLES}

The solution is an exact analytical solution to the Burgers equation for multiple frequencies and can be expressed explicitly in analytical form for every set of parameters. For numerical calculations, it is faster to use a recursion algorithm instead of using the Bessel functions directly. The result is valid for all distances as soon as the coefficients $c_{k}$ are calculated. The only problem is caused by limited representation of numbers in computers which results in errors for small $\epsilon$ as the series are converging slowly. The Cole-Mendousse solution has exactly the same problem when the dissipation is small. The time spent doing calculations is for 2 frequencies about 4-5 seconds on a Macintosh G3. For 200 frequencies it takes some minutes.

The first example is the specific situation when the difference frequency is the lowest frequency and also the same as one of the original frequencies: $V_{0}=\sin (\theta+$ $\phi)+\sin 2 \theta$ with $\epsilon=0.05$. Even though not used in the numerical calculations, the specific expression for the coefficients $c_{k}$ is given as an example of the explicit form of the coefficients:

$$
c_{k}=\sum_{n=-\infty}^{\infty}(-1)^{k-n} \mathrm{e}^{i(k-2 n) \gamma} I_{k-2 n}\left(\frac{1}{2 \epsilon}\right) I_{n}\left(\frac{1}{4 \epsilon}\right)
$$




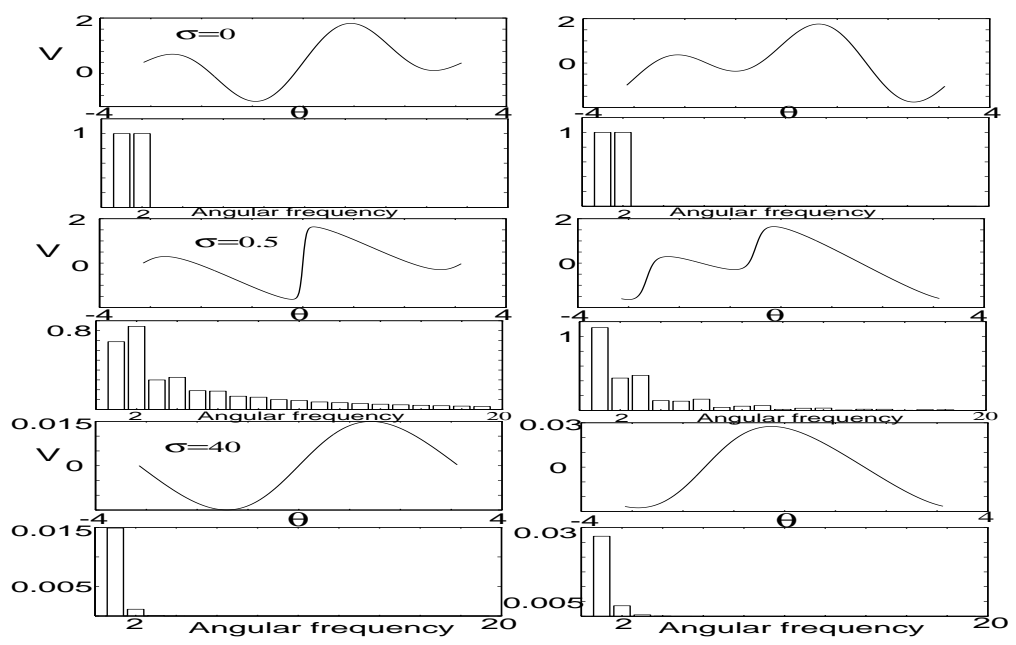

FIGURE 1. One of the original frequencies is the difference frequency and equal to the lowest frequency. Different phases affect the wave spectra. $V_{0}=\sin (\theta+\phi)+\sin 2 \theta, \epsilon=0.05$. Left: $\phi=0$. Right: $\phi=\pi / 2$.

In Figure 1 is seen the evolution for the phase $\phi=0$ in the left column, and the phase $\phi=\pi / 2$ in the right column. To begin with, at $\sigma=0$, they of course have the same frequency content. At the distance $\sigma=0.5$ is seen how the frequency 1 is larger when the phase $\phi=\pi / 2$ and has increased its amplitude to above its original one. The frequency 2 on the other hand is larger when the phase $\phi=0$, although it is not above its original amplitude. At a larger distance $\sigma=40$, the frequency 1 is twice the size when the phase $\phi=\pi / 2$. Surprisingly enough, also frequency 2 is larger. It is approximately three times as large as for when the phase $\phi=0$. This is due to the nonlinear transfer of energy from frequency 1 to its first higher harmonic, frequency 2 .
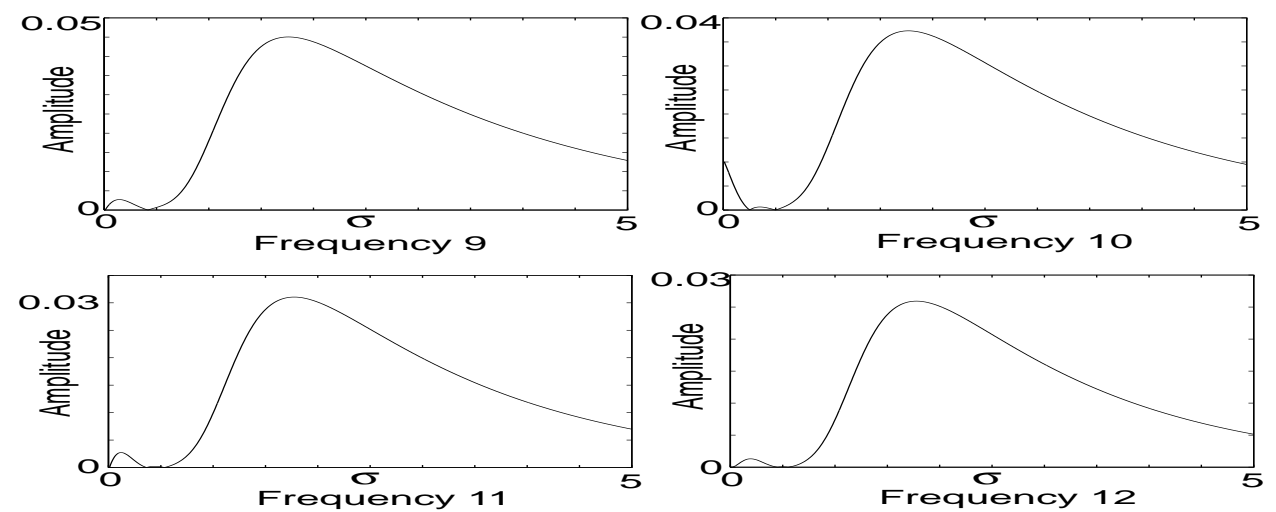

FIGURE 2. The oscillatory behavior of the high frequencies with small amplitudes in the presence of a strong low frequency. $V_{0}=\sin \theta+0.01 \sin 10 \theta \epsilon=0.05$.

In the next example, Figure 2, is shown a high frequency with small amplitude in 
the presence of a low frequency. The boundary condition is $V_{0}=\sin \theta+0.01 \sin 10 \theta$. The oscillatory behavior is seen not only for the frequency 10 but also for the surrounding components, in particular frequency 11 which has first one big and then one small oscillation. This disappears when the higher harmonics emanating from frequency 1 grows large enough to overwhelm the smaller contributions from the original frequency 10.

There is no theoretical limit on the number of frequencies. The limit on the computer used for obtaining the data in the figures about 220 frequencies, which is due to memory restrictions. As shown in the pulse in Figure 4, more or less any shape of pulse might be represented as long as the pulse fits within the chosen interval. The input must still be represented in the form of a sum of sine waves.
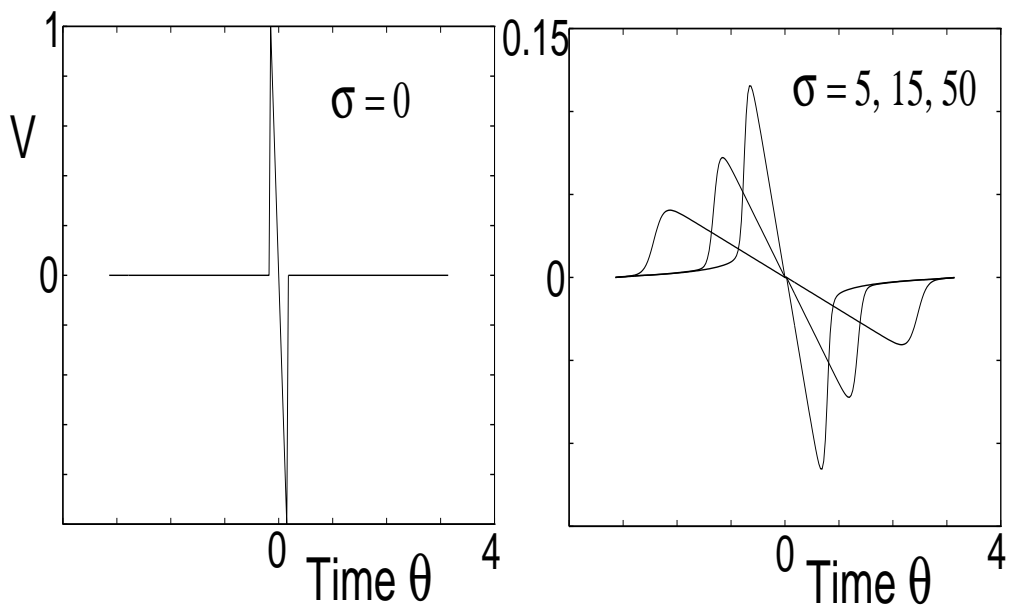

FIGURE 3. An initial N-wave and its evolution according to the multi-frequency solution.

\section{REFERENCES}

1. C.M. Hedberg, accepted for publication in J.Acoust.Soc.Am. in August 1999.

2. J.D. Cole, Quart. Appl. Math. 9, 225-236, (1951)

3. J.S. Mendousse, J.Acoust.Soc.Am. 25, 51-54, (1953)

4. F.H. Fenlon, J.Acoust.Soc.Am. 51, 284-289, (1972)

5. F.H. Fenlon, J.Acoust.Soc.Am. 53, 1752-1754, (1973) 